\title{
OSTEOCONDROSIS, FISITIS, DEFORMACIONES ANGULARES Y FLEXURALES EN EQUINOS COMO EJEMPLOS DE ENFERMEDADES ORTOPÉDICAS DEL DESARROLLO
}

\section{OSTEOCHONDROSIS, PHYSITIS, ANGULAR AND FLEXURAL DEFORMATION IN HORSES AS EXAMPLES OF DEVELOPMENTAL ORTHOPEDIC DISEASES}

\author{
Christian Tuemmers*, Carolina Mora, Angélica Saldivia \\ Universidad Católica de Temuco, Facultad de Recursos Naturales, Escuela de Medicina Veterinaria, Casilla 15-D, \\ Temuco, Chile. \\ *Autor para correspondencia: ctuemmers@uct.cl
}

Resumen

Las Enfermedades Ortopédicas del Desarrollo (EOD) son aquellas que afectan al aparato locomotor de los equinos jóvenes, las cuales tienen importantes consecuencias tanto en el rendimiento deportivo y reproductivo del animal. Estas patologías poseen un alto grado de incidencia dentro de los Haras en todo el mundo, provocando preocupación dentro del mercado equino, ya que es un factor que merma negativamente la economía de los criadores. La osteocondrosis, osteocondritis disecante, displasia fisiaria, deformaciones angulares y flexulares de los miembros se encuentran entre las patologías que se agrupan bajo esta terminología. Un factor común de estas patologías son las alteraciones en la osificación endocondral, ya sea en las placas de crecimiento metafisiarias o en las epifisiarias del aparato locomotor. El conjunto de enfermedades posee una sintomatología típica que incluye algún grado de claudicación, alteración en la postura, miembros encorvados y efusión articular. La patogénesis de estas enfermedades se basa principalmente en factores nutricionales, genéticos, mecánicos, de conformación o una combinación de estos. La nutrición aparece como un factor que puede ser controlado por los criadores para disminuir la incidencia de estas patologías, a través de la implementación de programas alimenticios que establezcan los niveles necesarios de energía, minerales y proteínas para las yeguas gestantes y los potros en proceso de crecimiento.

Palabras claves: enfermedades ortopédicas del desarrollo, equino, nutrición.

\begin{abstract}
Developmental Orthopedic Diseases (DOD) are those that affect the musculoskeletal system of young horses, which have important consequences both in sports and animal reproductive performance. These diseases have a high incidence within Equine Stud farms worldwide, causing concern within the equine market as it is a factor that undermines the economy negatively breeders. Osteochondrosis, osteochondritis dissecans, physeal dysplasia, angular deformities and flexulares members are among the diseases that are grouped under this terminology. A common factor of these diseases are disturbances in endochondral ossification, either metaphyseal plates in the epiphyseal growth or locomotor. The disease has a set of typical symptoms that include some degree of lameness, abnormal posture, hunched members and joint effusion. The pathogenesis of these diseases relies primarily on nutritional, genetic, mechanical forming or a combination thereof. Nutrition appears as a factor that can be controlled by breeders to reduce the incidence of these diseases, through the implementation of food programs to establish the necessary levels of energy, minerals and protein for pregnant mares and foals in process increase.

Keywords: Developmental Orthopedic Diseases, equine, nutrition.
\end{abstract}


Introducción

El deporte ecuestre presenta un sinnúmero de problemáticas relacionadas con la salud y el rendimiento deportivo del caballo, las cuales contrarrestan las ganancias acarreando cada año enormes pérdidas económicas para los criadores y propietarios. Estas actividades deportivas dependen mayoritariamente de los criadores, de las asociaciones de razas equinas existentes y de su mercado, en el cual la oferta y demanda está adscrita a la disciplina deportiva y la calidad del animal (Harris, 2005). Por tanto, la salud del equino es un elemento de gran relevancia, lo cual hace necesario profundizar en el conocimiento de su organismo, las patologías que lo aquejan y los métodos capaces de disminuir la incidencia o contrarrestar los daños de ciertas enfermedades (Gandarillas, 2008).

Las enfermedades ortopédicas del desarrollo representan un gran problema para el aparato músculoesquelético de los equinos, ya que pueden terminar su carrera deportiva (Reca, 2005). Dichas patologías tienen en su mayoría como factor común alteraciones en la osificación endocondral, tanto de las placas de crecimiento metafisarias como epifisarias (Baxter y Turner, 2004). Esta terminología fue utilizada por primera vez en el año 1986, en el congreso de la American Quarter Horse Association (AQHA) con el fin de abarcar todos los problemas ortopédicos observados en potros en crecimiento (Mcllwraith, 2004). La osteocondrosis, osteocondritis disecante, displasia fisiaria, deformaciones angulares y flexulares de los miembros son algunas de las patologías que se incluyen dentro de las "Enfermedades Ortopédicas del Desarrollo" (EOD).

Datos epidemiológicos muestran que la incidencia de EOD en la población equina mundial se presenta entre 10 a 26\% (Stephen, Jackson y Pagan, 2007). Este porcentaje reafirma la preocupación existente en el medio del deporte ecuestre en relación a las pérdidas económicas subyacentes al negocio equino. En el caso chileno, las cifras difieren bastante, ya que estas enfermedades sólo se presentan en un $5 \%$ en los equinos, esto según un estudio retrospectivo realizado en el hospital equino de la Universidad Austral de Chile, basado en casos atendidos entre 1976 y 1997. La diferencia porcentual estaría dada en razón al bajo nivel de investigación y estudios de prevalencia de estas patologías en Chile, además de la baja afluencia de los criadores de caballos a una atención especializada (Matamala, 1999).

Según algunos estudios, en los cuales se utilizaron las dosis recomendadas por la National Research Council (NRC), el aumento en la dosis de energía a un 129\% demostró que la sobreingesta de alimentos energéticos produce un aumento transitorio en los niveles circulatorios postprandiales de triyodotironina (T3), con una subsiguiente disminución de la concentración de tiroxina (T4), hormona responsable del proceso de maduración normal de los condrocitos (Ralston, 2007). Este tipo de fallas hacen posible la aparición de desórdenes en la osificación normal de los huesos y la consiguiente presentación de alguna EOD (Garzón A, Garzón R. y Martínez, 2008).

Además del desequilibrio energético, aquellos de carácter mineral son también de gran relevancia. Los desequilibrios minerales, como deficiencias de calcio, fósforo, cobre y zinc, son importantes en el proceso de maduración ósea normal con directa relación en el metabolismo óseo, ya que son constituyentes esenciales de metaloenzimas involucradas en la síntesis de cartílago y en el proceso de calcificación (Clutterbuck, Harris, Allaway y Mobasheri, 2010).

En base a lo anterior, el control de la dieta alimenticia en los equinos es de vital importancia, por lo que se hace necesario conocer adecuadamente los requerimientos que el animal necesita de acuerdo a la etapa de desarrollo que atraviesa. La atención debe estar en los niveles energéticos y la fuente que lo suministra, además de los niveles minerales (Pagan, 2003).

\section{Histofisiología de la osificación endocondral}

Un factor común presente en las anomalías conocidas como enfermedades ortopédicas del desarrollo (EOD), es que todas presentan algún grado de alteración o falla en la osificación endocondral, ya sea en las placas de crecimiento metafisarias o epifisarias (Baxter y Turner, 2004).

El proceso de formación del esqueleto de los potrillos comienza ya al primer mes de gestación (Baxter y Turner, 2004). Al principio se observa un pequeño fragmento diferenciado de cartílago hialino que comienza a cambiar gradual y progresivamente a hueso a través de un proceso denominado osificación endocondral (Mericq, 2007). La osificación endocondral, en términos simples, consiste en el reemplazo del cartílago hialino por hueso. Este mecanismo se inicia en los denominados centros de osificación primarios, ubicados en la mitad de la diáfisis de 
los huesos largos (Garzón et al., 2008). De forma secuencial, los principales eventos que se presentan en los centros primarios de osificación comienzan con la formación de un modelo de cartílago hialino del futuro hueso a formar, y paulatinamente este modelo comienza a crecer. Posteriormente, las células que conforman este molde, denominadas condrocitos, comienzan a hipertrofiarse dando por resultado la formación de lagunas. Paralelo a esto, la zona media del futuro hueso se vasculariza, lo que da pie a que células condrogénicas se conviertan en células osteoprogenitoras, que darán paso a la formación de osteoblastos los cuales producirán la transformación de cartílago en hueso por medio de la secreción de matriz ósea y la creación de un collar óseo en la superficie del molde del cartílago. Esto impide el traspaso de nutrientes a los condrocitos hipertrofiados, lo cual produce la muerte de ellos y la formación de lagunas que serán rellenadas posteriormente por hueso por parte de los osteoblastos (Garner y Hiatt, 2002).

De esta forma, la secuencia de eventos culmina en el momento que se reemplaza completamente el cartílago de la diáfisis por hueso, exceptuando las placas de crecimiento epifisarias o fisis ubicadas en cada extremo del hueso, las cuales continúan creciendo por medio de los centros secundarios de osificación. Dichos centros presentan un mecanismo similar al producido en la diáfisis, pero a la vez difieren ya que en este proceso no se forma un collar óseo que impida el traspaso, sino que comienzan a internarse células osteoprogenitoras que invaden el cartílago de la epífisis, las cuales se diferencian posteriormente en osteoblastos. Estos comienzan a secretar matriz en el esqueleto de cartílago con el objetivo de reemplazar completamente el cartílago epifisario por hueso, excepto en la superficie articular donde dicho cartílago persiste durante toda la vida del animal y en las placas de crecimiento epifisario o también denominadas fisis, principales encargadas del crecimiento longitudinal de los huesos largos después del nacimiento (Garner y Hiatt, 2002). Estas placas de crecimiento presentan una arquitectura celular característica que va desde el nacimiento hasta cuando cesa el crecimiento, momento en el cual estas fisis se osifican completamente, evento que varía con cada hueso (Baxter y Turner, 2004).

\section{Osteocondrosis}

Enfermedades ortopédicas del desarrollo en equinos de deporte

Características clínicas: la osteocondrosis (OC) se describe como una falla en los cartílagos de crecimiento que se produce tanto en animales como en seres humanos (Ytrehus, Carlson y Ekman, 2007). Esta patología se ha descrito en diferentes especies de animales entre las que encontramos los perros, gatos, ratas, cerdos y vacas, siendo los animales de rápido crecimiento los más susceptibles a presentar OC (Thompson, 2007). Las estimaciones más realistas sobre la OC establecen una prevalencía que oscila entre un 5 y $20 \%$, afectando a las principales razas de caballos (Peralta y Batalla, 2009). Estos datos no se alejan de la realidad chilena que reporta un $5 \%$ de equinos con OC de un total de 237 ejemplares dentro del grupo con afecciones articulares (Matamala, 1999).

En la actualidad, la OC es reconocida como una causa frecuente de claudicación en caballos jóvenes de deporte (Ytrehus et al., 2007), produciendo importantes pérdidas económicas en la industria equina mundial. En base a estudios realizados por Dr. Rene Van Weeren, cada año en el noroeste de Europa unos 20.000 potros se ven afectados por alguna forma de OC (Peralta y Batalla, 2009), desarrollándose durante los primeros meses de vida del animal (Van Weeren, 2006). Sin embargo, puede que no se evidencien signos clínicos hasta que el caballo comience su entrenamiento o incluso en su estado adulto (Novales, 2008). Por años los términos osteocondrosis, osteocondritis y osteocondritis disecante se han utilizado como sinónimos, sin embargo estos términos se han diferenciado ya que la osteocondrosis se refiere específicamente a la enfermedad, la osteocondritis es la inflamación que se produce en la osteocondrosis y la osteocondritis disecante es cuando a esta inflamación producida se le agrega un desprendimiento o colgajo de cartílago dentro de la articulación (Baxter y Turner, 2004). Las lesiones osteocondróticas en los caballos se pueden presentar en cualquier parte del cuerpo (Carlson, Cullins y Meuten, 1995). Sin embargo existen varios sitios donde se presentan con mayor frecuencia, entre los que encontramos la cresta tróclear lateral y el cóndilo medial del fémur, la rótula, el borde dorsal de la cresta sagital de la tibia y el tarso (Wittwer, Hamann y Rosenberger, 2006).

Propuestas etiológicas y fisiopatológicas: en la actualidad, la etiología y la patogénesis de la OC no se encuentran del todo claras y han sido objeto de un reciente debate por parte de la comunidad científica. Actualmente han surgido una serie de pistas a partir de estudios realizados en cerdos, caballos y perros que han determinado que la etiología es de carácter multifactorial (Semevolos y Nixon, 2007). Es probable que se involucren factores del tipo traumáticos o biomecánicos en el cartílago los cuales se han visto debilitados por desequilibrios nutricionales $u$ hormonales, trastornos vasculares o factores genéticos. Cualquiera sea la causa, la lesión inicial parece estar dada por un daño isquémico presente en el cartílago de crecimiento (Ytrehus, Carlson y Ekman, 2007). 
En los caballos adultos y en los diferentes animales la difusión de nutrientes en el área intraarticular se realiza a través del líquido sinovial ya que el cartílago articular es avascular, a diferencia de lo que pasa en los caballos jóvenes donde esta nutrición depende exclusivamente de vasos sanguíneos viables, los cuales con el tiempo comienzan poco a poco a desaparecer a través de un proceso denominado condrificación del cartílago articular (Carlson et al., 1995). Investigaciones realizadas en potrillos revelan que los vasos sanguíneos que nutren al cartílago articular de la epífisis distal de la tibia comienzan a desaparecer ya a los 3 meses de edad y que para el caso de los cóndilos femorales esto se produce a los 5 meses de edad (Carlson et al., 1995). Observaciones sugieren que existiría un periodo de riesgo durante el cual el cartílago epifisario depende del suministro sanguíneo. Interrupciones durante este periodo en animales jóvenes de rápido crecimiento podrían dar pie a la formación de OC (Foerner, 2003). Esto es gracias a la isquemia provocada por este tipo de interrupciones, ya que se producen focos necróticos de cartílago con la subsiguiente falla en el proceso de osificación endocondral (Carlson et al., 1995). Cuando este tipo de lesiones se desarrollan en caballos muy jóvenes, con frecuencia se producen defectos suficientemente grandes, lo que forma fisuras en las capas profundas del cartílago necrótico, presumiblemente por una disrupción de capilares debido a fuerzas de deslizamiento (Baxter y Turner, 2004). Este tipo de fisuras presentes en el cartílago articular pueden extenderse desde el estrato necrótico profundo hasta la superficie del cartílago, dando pie a la formación de un colgajo o resto de cartílago (Ytrehus et al., 2007). Estos fragmentos una vez separados se osifican y pueden permanecer unidos al resto del hueso o quedar libres en el interior de la articulación. Si estos fragmentos se desprenden pueden causar daño y erosión sobre las superficies articulares, acompañado esto de un proceso inflamatorio en la membrana sinovial. Esta etapa avanzada de la OC es denominada como osteocondritis disecante (Baxter y Turner, 2004).

La OC además de participar potenciando la aparición de osteocondritis disecante en el caballo, también se ha indicado como factor predisponente en el desarrollo de quistes óseos subcondrales (Carlson et al., 1995), los cuales se encuentran comúnmente en la cara distal del cóndilo femoral medial, cara distal del metacarpo, carpo, codo, y falange (Ytrehus et al., 2007). Se sugiere que estos quistes subcondrales podrían desarrollarse a partir de invaginaciones del cartílago articular provenientes de restos de necrosis, lo cuales son producidos en el complejo cartilaginoso articular-epifisario (Baxter \& Turner, 2004), o en respuesta a una fuga de fluido sinovial en el hueso subcondral producto de defectos en el cartílago articular (Ytrehus et al., 2007). Por medio de estudios experimentales en potrillos se han reproducido este tipo de lesiones a través de un daño traumático sobre el cartílago articular, lo que sugiere que no todos los quistes subcondrales se encuentran relacionados con la OC (Carlson et al., 1995).

Diagnóstico: hasta la fecha, el diagnóstico de la OC se basa principalmente en los signos clínicos presentes en el animal, esto siempre acompañado de estudios radiográficos. Recientemente, investigaciones en el área de biomarcadores relacionados con la OC han abierto una nueva propuesta de diagnóstico (Van Weeren, 2006).

Uno de los datos de anamnesis importantes desde el punto de vista diagnóstico de la enfermedad es la edad del animal, ya que este tipo de patologías se presenta principalmente en animales jóvenes con presencia de efusión articular, el cual puede ser unilateral o bilateral afectando principalmente las articulaciones tarso tibial, femoropatelar, o metacarpofalángicas, evidenciando algún grado de claudicación (Baxter y Turner, 2004). Los casos más graves se presentan a temprana edad siendo la cresta tróclear lateral del fémur la de mayor prevalencia, evidenciando en la mayoría de estos potros una claudicación severa (Cruz, 2007). Por otro lado manifestaciones de esta enfermedad comienzan a aparecer al inicio del entrenamiento de los equinos, presentándose comúnmente a los 3 o 4 años de edad. Todo esto dependerá de la raza y la aptitud deportiva que presente. En casos excepcionales, la presencia de manifestaciones clínicas puede comenzar a evidenciarse incluso en animales sobre los 10 años. En la mayoría de estos casos, estas manifestaciones tardías se asocian con algún cambio en el su entrenamiento (Van Weeren, 2006). En el examen radiográfico, en estos sitios de mayor incidencia de este tipo de patologías el hallazgo de fragmentos sueltos, aplanamiento o irregularidades en la superficie de la articulación dan cuenta de la presencia de OC en la articulación afectada (Van Weeren, 2006).

Tratamiento: en el tratamiento de la OC existen básicamente dos métodos. Primero se encuentran los de carácter conservador, los cuales consisten en el reposo del animal afectado, ejercicios controlados y la aplicación de antiinflamatorios no esteroidales (AINES). Este tipo de tratamientos tiene un alcance terapéutico limitado y dependerá mucho del grado de afección que el animal presente (Baxter y Turner, 2004).

Para casos más complicados existe la intervención quirúrgica como tratamiento de elección más apropiado. Uno de los puntos importantes al momento de elegir este método terapéutico es la edad del animal, ya que el carácter dinámico que presenta la OC durante los primeros meses ( 8 a 9 meses), debido a la alta tasa de 
recambio que sufre el colágeno articular, puede llegar a producir curaciones espontáneas. Por lo cual antes del año de edad se recomienda no realizar este tipo de intervenciones ya que puede ser innecesaria (Thompson, 2007).

La técnica de elección más utilizada en la actualidad es la artroscopia utilizada en todas las articulaciones afectadas, excepto en las articulaciones vertebrales cervicales donde este tipo de cirugías no se realizan. La técnica en términos simples consiste en la penetración de la articulación afectada hasta la porción de hueso subcondral, el cual se perfora favoreciendo la llegada de vasos sanguíneos y factores de crecimiento plaquetarios desde la medula ósea. Se realiza un lavado de la articulación retirando todos los fragmentos de cartílago desprendidos en el procedimiento (Cruz, 2007).

El pronóstico de este tipo de lesiones varía dependiendo del sitio de la lesión, pero por lo general hablamos de un pronóstico de bueno a excelente.

\section{Deformaciones angulares de los miembros}

Las deformaciones angulares de los miembros es una patología incluida en las EOD siendo mundialmente diagnosticada y tratada desde hace muchos años (Silva, 2005).

Características clínicas: las deformaciones angulares de los miembros se definen como una desviación del miembro locomotor del eje normal, en un plano frontal y existen dos tipos de desviación. Por un lado tenemos el valgo, en el cual la extremidad se desvía hacia lateral, distal al punto de la deformidad. Por otro lado está el varo, el cual consiste en la desviación del miembro hacia medial, en ventral de la deformidad (Barr, 1995).

\section{Propuestas etiopatológicas y fisiopatologícas}

Herencia/ predisposición genética: según Mitten y Bertone (1994), existen tres mecanismos que pueden originar el desarrollo de esta patología. El primero se refiere a la laxitud del soporte ligamentoso periarticular que durante la gestación no sufre ningún esfuerzo. Este defecto normalmente se corrige en los primeros días de vida producto del ejercicio que empieza a realizar el potrillo (Bertone, 2004). También se considera como mecanismo originador el crecimiento asincrónico de las fisis de los huesos largos. Estas pueden producirse durante la gestación o en los primeros meses de vida. Lo que ocasiona el crecimiento dispar de los huesos es el desarrollo asincrónico del periostio y la atrofia de los precursores cartilaginosos. También puede presentarse un desarrollo deficiente de los huesos cortos del tarso y/o carpo (Barr, 1995). Otros factores que generan esta anomalía son los defectos congénitos producto de una mala posición intrauterina del feto.

Estrés mecánico y trauma: las placas de crecimiento pueden verse afectadas por varios factores que producen asimetrías en el desarrollo. Uno de estos factores son los traumas o ejercicios excesivos que generan una compresión de la zona proliferativa del hueso, siendo esta la mayor causal de deformidad. Si este trauma es severo ocasiona isquemia y cierre de un lado de la fisis, mientras que el lado opuesto sigue creciendo (Auer et al., 1982). Los potrillos con claudicación o dolor en un miembro pueden presentar deformaciones angulares en el miembro opuesto por los efectos del apoyo.

\section{Diagnostico}

Para llegar a un diagnóstico acertado de debe tomar en cuenta la historia clínica del animal, en donde son factores de relevancia las condiciones de nacimiento del potro, si es que éste nació prematuro. Se debe tener en cuenta cuándo se detectó el problema, cómo ha evolucionado, cual fue la alimentación de la madre en el último tercio de la gestación y la del potrillo. Es importante conocer las características nutricionales de la madre, si es que hubo sobrepeso al momento del parto y si el potro tiene algún tipo de claudicación en el miembro. Luego de tener esta información se debe dar paso al examen físico, en donde se buscarán datos como el sitio de deformación, el tipo de desviación, el grado de severidad de la deformación y si es que esta puede ser corregida por presión manual o no. Junto con esto se debe tener conocimiento de la existencia de claudicación, de dolor, inflamación o tumefacción (Wyn- Jones, 1992).

Como en todas las EOD, la evaluación radiográfica es un punto de importancia. Esta debe ser dorsopalmar y lateromedial para el miembro torácico, latero medial y caudo craneal para el miembro pelviano. Este método es una forma confiable de conocer el sitio y el ángulo exacto de la desviación (Barr, 1995). 
El tratamiento depende de los mecanismos que originaron la desviación, del tipo, del grado de la misma, y de la edad del potrillo en la que se haya detectado el problema. Estos mecanismos se dividen en tratamientos conservadores y quirúrgicos.

Los tratamientos quirúrgicos tienen como objetivo alinear la porción proximal del miembro afectado, además de restituir el normal paralelismo de las superficies articulares con el suelo. Entre las técnicas útiles se encuentran la resección del periostio. El principio de este procedimiento se basa en la sección de periostio en forma de (T) invertida, un centímetro por encima de la placa de crecimiento (Gaughan, 1998). Esta cirugía se realiza en potrillos con desviaciones angulares producto de un crecimiento dispar de los huesos largos, en los que la deformación es grave y no respondió al tratamiento conservador. Se basa en la estimulación del desarrollo óseo del lado cóncavo de la deformación, para igualar al lado opuesto (Barr, 1995).

Otra técnica es el uso del tornillo transfiseal, indicado en casos de crecimiento dispar de los huesos largos en potrillos jóvenes. Se basa en la colocación de un tornillo en la fisis del hueso afectado, del lado de mayor crecimiento óseo, correspondiente al lado lateral, en una desviación varo y al lado medial en una desviación valgo (Wyn- Jones, 1992).

Deformaciones flexulares de los miembros.

Las deformaciones flexulares de los miembros son patologías que hacen referencia a un acortamiento o hiperextensión que dificulta el funcionamiento normal de músculos y tendones flexores. Esto impide que el miembro locomotor del potrillo adopte una posición normal, lo cual provoca una desviación hacia craneal o caudal, en un plano sagital (Wyn- Jones, 1992).

Anteriormente, las deformidades flexulares se denominaban retracciones tendinosas o contracturas flexulares. Las deformaciones flexurales de los miembros se pueden clasificar en congénitas o adquiridas. Aunque es frecuente agrupar ambos síndromes juntos ya que los dos producen una desviación de los ejes óseos, es muy probable que sean dos entidades separadas. En ambos síndromes, los mecanismos son multifactoriales y de naturaleza especulativa (Adams y Santschi, 2000).

\section{Propuesta etiopatológica y fisiopatológica}

Existen múltiples factores que son responsables del origen de estas patologías que deforman los miembros. Estos pueden ser diferenciados al igual que el resto de las anomalías estudiadas en el presente trabajo, en genéticos, nutricionales, mecánicos y de conformación.

El factor externo de mayor relevancia que influye sobre la normal diferenciación de los componentes articulares es el movimiento del animal, por lo cual el estrés mecánico y trauma forman parte de los factores desencadenantes de las deformaciones flexurales. Sin el movimiento se produce una regresión del desarrollo, fusión y anquilosis fibrosa. En el caso que un animal tenga un miembro completamente inmóvil no existe tratamiento útil y el mantenimiento de un animal con estas características puede producir deformaciones severas (Wyn- Jones, 1992). Además de esto, el dolor que el animal siente en un miembro provoca reflejos de retirada y esto disminuye las fuerzas de apoyo sobre el pie del miembro comprometido.

Las deformaciones flexulares en los miembros del animal devienen también de una predisposición genética aunque igual que en el resto de las EOD, estas son desconocidas (Trotter, 2006).

\section{Características clínicas}

Patologías congénitas: deformación flexural de la articulación metacarpo/ metatarso falangiana: La hiperflexión leve de esta articulación se presenta con más frecuencia en potrillos recién nacidos. Esta deformación a veces puede corregirse manual o espontáneamente por correcta relación entre los músculos y los tendones agonistas y antagonistas. En el caso que no se corrija bajo esos términos, es necesario aplicar tratamientos de fisioterapia o la colocación de férulas.

Deformación flexural del carpo: Son anomalías poco comunes, generalmente graves y de presentación bilateral. Cuando es una anomalía leve se observa una convexidad hacia craneal del carpo y se puede corregir manualmente con ayuda de férulas y ejercicio controlado. La cirugía sólo es recomendable cuando los tratamientos conservadores no entreguen resultados (Adams y Santschi, 2000). 
Deformación flexural de la articulación interfalangiana proximal y distal: Es poco frecuente en potrillos recién nacidos pero cuando se presenta abarca los músculos y tendones flexores digitales superficiales y profundos. El animal presenta dificultad para apoyar el talón en el suelo, aun así, si logra ponerse en pie y ofrece posibilidades de corrección manual, su pronóstico es favorable. Como en las otras malformaciones, si el caso es severo, la cirugía es la alternativa que sigue a los métodos conservadores (Almanza, 2008).

Flacidez de los tendones flexores: Es una anomalía bastante frecuente en los potros recién nacidos que afecta los miembros pelvianos pero puede comprometerlas cuatro extremidades. Este problema está asociado a la falta de ejercicio y su corrección es posible en los primeros días luego del nacimiento. El signo clínico del animal que sufre la anomalía es que este camina sobre la superficie palmar/ plantar del casco, evitando apoyar las pinzas (Barr, 1995).

Hiperextensión digital: Afecta a potros recién nacidos pero es de poca incidencia. Se presenta como una flacidez tendinosa extrema. Su signo clínico muestra un potrillo que rebaja demasiado sus talones provocando un exceso de presiones en esta región lo cual compacta la almohadilla plantar y, en consecuencia, permite que el impacto del piso repercuta en la articulación interfalángica distal y el aparato podotroclear del pie (Wyn- Jones, 1992).

Patologías adquiridas: deformidad flexural del carpo: se relaciona con la ruptura del músculo del tendón extensor digital común. Sus signos se manifiestan a través de una leve flexión a nivel del carpo y presencia de tumefacción en la cara craneolateral de dicha articulación.

Deformidad flexural de la articulación metacarpo falángica: Es una patología que se presenta en animales cercanos al primer año de edad. En este caso, el hueso que posee crecimiento acelerado es el radio, con respecto al tendón del músculo flexor digital superficial. Este tendón se genera en la metáfisis del radio, a través del ligamento frenador supracarpiano, fibroso e inelástico, que puede considerarse como la cabeza radial del músculo, y se inserta en la primera y falange intermedia. El crecimiento acelerado durante los períodos de buena nutrición, sobrepasa a la capacidad del ligamento frenador para extenderse, de ésta forma, para compensar la falta de longitud del tendón, la articulación MCF, adopta un estado de progresiva flexión. Este tipo de patologías en general es de presentación bilateral (Wagner, Watrous, Kaneps, Schmotzer y Riebold, 1985). El tratamiento es de urgencia, si la articulación MCF se mantiene en flexión por un tiempo prolongado pueden verse afectados, el músculo interóseo III (ligamento suspensorio); el ligamento frenador infracarpiano; la superficie caudal de la articulación MCF y las adherencias paratendinosas del músculo del tendón del músculo flexor digital superficial, provocando que estas estructuras se acorten o dejen de crecer. También pueden verse afectadas la superficie craneal de la articulación y el tendón del músculo extensor digital común, el cual tiende a estirarse demasiado (0’Grady, 2006). El tratamiento en los casos leves puede ser efectivo a través del herraje correctivo. La cirugía está indicada en los casos más severos y consiste en la técnica de desmotomía del ligamento frenador supracarpiano (Trotter, 2006).

Deformidad flexural de la articulación interfalángica distal: Esta patología se manifiesta antes del destete, presenta la falange distal flexionada en relación con la falange intermedia. Al progresar la enfermedad, el cuadro puede presentar un ángulo mayor de $90^{\circ}$ en relación a la cara dorsal de la muralla y el suelo. A todo esto se suma el desgaste de las pinzas que puede tener como consecuencia una infección bilateral del casco (Wagner et al., 1985).

Diagnóstico: el historial clínico del animal es relevante para la identificación del origen congénito o adquirido de la deformación, para la definición de la fecha de detección del problema y su evolución (Adams y Santschi, 2000).

Tratamiento: los tratamientos de las deformaciones flexulares de los miembros se clasifican en conservadores, y quirúrgicos. Entre los tratamientos conservadores se encuentran los ejercicios controlados, el uso de férula, desvasado correctivo, botas ortopédicas, entre otros. Entre los tratamientos quirúrgicos se destaca la desmotomía del ligamento frenador distal. La elección del tratamiento depende del grado de severidad de la deformación, del tipo de deformación flexural y del momento en el que se diagnostica el problema (Wagner et al., 1985).

$$
\text { Displasia fisaria (fisitis) }
$$

Estas patologías se presentan principalmente en animales de edades que fluctúan entre los 4 y 8 meses de edad. A pesar de ello potrillos de 1 y 2 años también pueden desarrollarla.

Baxter y Turner (2004) señalan que las terminologías "fisitis" o "epifisitis" pueden ser erradas o mal interpretadas ya que no se presenta un proceso inflamatorio activo de la fisis, epífisis o metáfisis de las piezas 
óseas comprometidas. Por tanto, la denominación de "displasia fisaria" sería la más acertada debido a que se trata de una anomalía que compromete el desarrollo del proceso de osificación, es decir, se está frente a una interrupción de dicha osificación endocondral dentro del cartílago de crecimiento fisario.

Esta alteración ósea posee un componente mecánico que se ve acentuado por la compresión aplicada a la fisis, de ahí que se conozca también como "compresión fisaria" (Baxter y Turner, 2004). Esta presión sobre la fisis provocaría un incremento de su espesor por el retraso de la calcificación provisional y además, un incremento de la supervivencia de los condrocitos. Este fenómeno manifiesta una acción de autocorrección del organismo del animal a través del cual se compensa cualquier pequeña angulación producida sobre el eje del miembro. A pesar de esto, si dicha compresión sobrepasa los límites fisiológicos de la fisis es probable que se origine una interrupción completa de la osificación endocondral.

Propuesta etiopatológica y fisiopatológica

La presencia de fisitis en los equinos puede responder a múltiples factores los cuales dependerán del caso clínico que se estudie. En aquellos casos en los cuales el animal presenta compromiso de varios miembros, es probable que el cuadro esté causado por problemas de índole nutricional. Por otro lado, cuando la anomalía compromete sólo una zona, se puede deducir que esto ha sido originado por un trauma o un exceso de compresión en la fisis afectada. Otros factores que pueden contribuir al desarrollo de enfermedades de este tipo son la predisposición genética, estrés mecánico por ejercicio inapropiado, obesidad, crecimiento demasiado acelerado y una nutrición desequilibrada o inapropiada (Harris, 2005).

Las diferentes combinaciones de los factores antes señalados, pueden estar involucradas en los diferentes casos de fisitis y otras enfermedades ortopédicas del desarrollo. Factores de carácter medioambiental o de manejo determinan si el potro desarrolla finalmente alguna enfermedad. Un buen control nutricional puede disminuir considerablemente el riesgo de sufrir EOD (Baxter y Turner, 2004).

Características clínicas: las principales zonas afectadas son las porciones distales del radio, la tibia y el tercer metacarpiano/metatarsiano (Baxter \& Turner, 2004), en estos casos se produce un acampanamiento de la metáfisis, produciendo un crecimiento notorio en estas articulaciones que provoca signos de dolor a la palpación y aumento de la temperatura.

Si nos referimos a la fisitis de la porción distal del metacarpo, ésta afecta generalmente a los cuatro miembros, y no simultáneamente a las porciones distales del radio y la tibia.

En cuanto a la OC y la fisitis que afectan las vértebras cervicales, pueden producir en el animal joven malformaciones en la columna vertebral, como el síndrome de Wobbler, también conocido como "síndrome del tambaleo" (Baxter y Turner, 2004).

Diagnóstico: el diagnóstico se basa generalmente en los signos clínicos como la inflamación y el aumento de la temperatura. Las radiografías son otro método y a través de ellas es posible observar más comúnmente una anormalidad denominada "labio fisario" la cual consiste en la producción de hueso parafisario, entre otras anomalías (Baxter y Turner, 2004).

Tratamiento: como primer paso para el tratamiento de la fisitis se debe evaluar la ración alimenticia del caballo. En el caso que se presenten múltiples animales afectados, se puede indicar que existe la posibilidad de una deficiencia nutricional geográfica. La ración del animal debe ser moderada según sea necesario y se aconseja reducir el peso corporal o la tasa de crecimiento. Muchas compañías de alimentos han desarrollado raciones equilibradas específicas para caballos en proceso de crecimiento con el objetivo de minimizar el desarrollo de fisitis y otras enfermedades ortopédicas del desarrollo. Según los autores (Thompson, 2007), no se conocen los resultados de estas disposiciones en las raciones alimenticias propuestas por las compañías de alimentos. En cuanto a los fármacos utilizados en el tratamiento de la fisitis, aquellos de carácter antiinflamatorio no esteroideos (AINES) están indicados en la mayoría de los casos a efectos de disminuir la inflamación fisaria y mejorar la marcha rígida del animal. Estos fármacos (AINE) ayudan a disminuir el dolor y prevenir el desarrollo de deformaciones flexulares. El uso de estos medicamentos puede ser necesario durante 2 a 4 semanas en bajas dosis. Tal es el caso de la fenilbutazona por vía oral en días alternos, la cual entrega buenos resultados (Baxter y Turner, 2004). Se debe tener en cuenta que las dosis no debieran ser altas ya que tiene como efecto colateral el riesgo de úlceras gástricas en el caballo cuando se indican por periodos largos. 
Los procedimientos que pueden aportar beneficios en favor de prevenir mayores traumas a la fisis serían la disminución del ejercicio (confinamiento) y la corrección de las causas predisponentes, tales como deformaciones angulares (Sellnow, 1999).

Los casos de fisitis leve son frecuentemente autolimitantes y se resuelven cuando el esqueleto madura y cesa el crecimiento de las fisis afectadas. Por otro lado, los casos más graves de fisitis que tienen anormalidades ortopédicas simultáneamente pueden ser causantes de problemas residuales de una importante gravedad como para limitar el rendimiento atlético futuro (Baxter y Turner, 2004).

\section{Factor nutricional y su importancia}

La revisión de las enfermedades ortopédicas del desarrollo del presente trabajo ha indicado que la patogénesis de las anomalías puede ser multifactorial, y dentro de los factores mencionados la dieta y la nutrición juegan un papel clave para su prevención.

Se indica que los desequilibrios energéticos, de carbohidratos y minerales causan mineralización anormal del hueso lo cual se traduce en una propensión a las anomalías en los miembros de los caballos. Antes se culpaba a las proteínas de estos desequilibrios nutricionales pero esto no parece ser cierto si se piensa que muchos pastos proporcionan un $20 \%$ de proteína de la materia seca. Es necesaria una dieta equilibrada para lograr la salud musculoesquelética del caballo ya que la desnutrición incluye tanto la subalimentación como la sobrealimentación de nutrientes. Ambas dietas desequilibradas pueden provocar daño permanente en los miembros y tener posibles repercusiones metabólicas (Ralston, 2007).

También se debe tener presente la importancia del equilibrio mineral y la relevancia de tomar en cuenta las diferencias regionales respecto a la presencia de microminerales como el selenio, cobre, zinc y magnesio. Esto significa que las raciones deben estar estrictamente relacionadas con el área geográfica y la disponibilidad de alimentos.

Investigaciones indican que en una dieta adecuada de la yegua gestante y su cría se sientan las bases para el desarrollo apropiado de los huesos (Pagan, 2003). Es en la juventud del caballo en donde se encuentra el peligro ya que el período desde que nace hasta los 18 meses de edad es crítico para el crecimiento de los potros.

Una de las formas propuestas para evitar la incidencia de las enfermedades ortopédicas del desarrollo es un programa adecuado de alimentación tanto de la yegua gestante como del potrillo, ya que una mala alimentación puede provocar problemas ortopédicos de por vida (Harris, 2005).

Una cantidad inadecuada de proteínas en la dieta de la yegua durante la preñez puede inducir hipotiroidismo en el feto, lo cual podría mantenerse luego del nacimiento, disminuyendo el peso al momento de nacer y ocasionando una falta de crecimiento permanente. Esto puede ir acompañado además de un retraso en la osificación de los huesos cuboides cartilaginosos del carpo y el tarso ya que este proceso se produce comúnmente entre las 6 y 8 semanas de gestación y los 33 días posteriores al parto. Cuando el potrillo es normal al nacer y durante las primeras semanas de vida se desarrollan anomalías en el aparato musculoesqueletico, antes de los 3 meses de edad, las causas pueden ser nutricionales, dentro de las que encontramos la ingesta inadecuada de leche o también producto de que la leche sea deficiente en su concentración de minerales (Harris, 2005). Existen tres etapas dentro de las necesidades nutricionales de la yegua gestante las cuales están basadas en las etapas de producción. La primera etapa se inicia en la concepción y se prolonga aproximadamente hasta los primeros siete meses de gestación. La segunda etapa abarca el último trimestre de la gestación, alrededor de los siete meses de gestación hasta el parto. Finalmente, la última etapa es la de lactancia, que dura entre cuatro meses y seis meses postparto. Los errores más comunes en la alimentación de las yeguas preñadas son la sobrealimentación durante el gestación y la falta de alimentación durante la lactancia (Pagan, 2003).

Los requerimientos de nutrientes de la yegua aumentan de manera considerable luego de parir. Durante los 3 primeros meses de lactancia, la producción de leche aumenta por día a una tasa igual a 3\%, en relación a su peso corporal. Esta leche debe ser rica en energía, proteína, calcio, fósforo y vitaminas, por lo cual la yegua debe ser alimentada lo suficiente para satisfacer el aumento de las necesidades nutricionales (Harris, 2005).

Al acercarse el parto, la ingesta de alimentos debe aumentar paulatinamente para que la yegua se encuentre consumiendo la cantidad necesaria de alimento, lo cual logrará que la producción de leche sea óptima. Además de esto es necesario que la yegua no aumente su ingesta de grano en los últimos momentos de la gestación ya que esto puede provocar laminitis y cólicos. Luego de los tres primeros meses de lactancia el nivel de 
producción de la leche comienza a disminuir, por tanto debe reducirse nuevamente la ingesta de alimentos para mantener la condición física del animal (Phillips, 2011).

En el caso de las crías, si la yegua ha sido alimentada de forma correcta no es necesario complementar la lactancia con minerales hasta llegar a los 90 días de edad, a los cuales se puede introducir e ir en aumento en un $0,5 \mathrm{~kg}$ por mes de edad. Es muy importante que la cría se acostumbre a comer grano antes del destete ya que al concluir este es muy posible que disminuya su tasa de crecimiento. Esto puede acarrear un brote de crecimiento compensatorio que tendría como resultado alguna enfermedad ortopédica del desarrollo (Pagan, 2003).

Para la prevención de enfermedades ortopédicas del desarrollo existe una etapa en el crecimiento del animal que es crítica, la cual parte desde el destete hasta los 12 meses de edad (o hasta los 18 meses según otros autores), momento en que el esqueleto es más vulnerable a la ingesta de nutrientes y enfermedades, por tanto el equilibrio de estos es de suma relevancia.

Al cumplir los 12 meses de edad, el potro tiene menos posibilidades de sufrir alguna enfermedad ortopédica del desarrollo que un animal aún más joven, porque muchas de las lesiones que toman relevancia clínicamente después se ha formado antes de los 12 meses. El equilibrio de los nutrientes a esta edad continúa siendo muy importante, además del retraso de la ingesta de energía con motivos comerciales, ya que aún el esqueleto del animal es vulnerable.

\section{Conclusiones}

La Osteocondrosis, fisitis, deformaciones angulares y flexurales de los miembros se encuentran entre las enfermedades ortopédicas del desarrollo que se presentan en equinos de deporte. La fenomenología de las lesiones osteocondróticas, obedece a alteraciones en el proceso de diferenciación celular del cartílago del crecimiento, esto es, alteraciones en la osificación endocondral que afectan al cartílago articular-epifisiario o a la placa de crecimiento metafisiaria. Defectos en la superficie de las articulaciones de orden genético o en respuesta a trauma en el cartílago articular y hueso subcondral, pueden ocasionar un repliegue anormal del cartílago que impide el ingreso de osteoclastos y vasos sanguíneos al hueso subyacente, el cual se necrosa dando lugar a una lesión quística, denominada quiste subcondral. Diversos mecanismos patogénicos, tales como crecimientos asincrónicos de las fisis óseas, infartos locales, laxitud ligamentosa y anquilosis fibrosa secundaria a defectos del desarrollo, entre otros, pueden ocasionar la desviación permanente de los de los miembros afectados, respecto de sus ejes óseos, esto es, deformaciones angulares y flexulares. Por su parte, la displasia fisaria es una condición debida al alargamiento de la placa metafisiaria y posterior compresión mecánica de la fisis, en forma secundaria a la interrupción proceso de osificación endocondral dentro del cartílago de crecimiento fisario y autocorrección compensatoria de la angulación, respectivamente.

El diagnóstico clínico presuntivo de una enfermedad ortopédica del desarrollo, se realiza sobre la base de los hallazgos de la anamnesis y examen físico. Claudicación, alteración en la postura, miembros encorvados y efusión articular, forman parte de la signología típica de una EOD. Sin embargo, esta signología no es patognomónica de ninguna EOD, más bien, es común a la mayoría de las enfermedades osteoarticulares y musculares. Por esta razón, para objetivizar el diagnóstico, es necesario complementar los hallazgos con imagenología. Dentro de este contexto, la radiología es la técnica más utilizada, no obstante, en la actualidad, se han realizado esfuerzos por implementar técnicas con mayor grado de especificidad, tales como el uso de biomarcadores, se tratan de compuestos que se liberan a la circulación a partir de la degradación de la matriz cartilaginosa y pueden detectarse mucho antes de que aparezcan los signos clínicos o cambios radiográficos, hasta la fecha este método diagnóstico se encuentra en proceso de investigación.

El exceso de energía en la dieta, asociada a estados sostenidos de hiperinsulinemia y el efecto promotor del cartílago metafisiario, secundario al aumento del tono de la hormona del crecimiento, redunda en crecimientos óseos anormales durante el desarrollo del caballo. Del mismo modo, un nivel de proteína por debajo de los requerimientos y/o deficiencias en el suministro de minerales, tales como calcio, fósforo, zinc, magnesio, y cobre, redundan en la ocurrencia y/o predisposición al desarrollo de EDO en forma secundaria a la alteración de la homeostasis hormonal. 
Sustainability, Agri, Food and Environmental Research 4(2), 2016: 1-12

ISSN: 0719-3726

Referencias

Auer, J. R. Martens, \& E. Morris. 1982. Angular limb Deformities in Foals, partCongenital factors. Compend Contin Educ Pract Vet. 4:330-339.

Adams, S. \& E. Santschi. 2000. Management of congenital and acquired flexural limb deformities. Procceding of the American Association. http://www.ivis.org/proceedings/AAEP/2000/117.pdf

Almanza, A. 2008. Deformaciones angulares y desviaciones flexurales en potrillos.

http://www.redboot.com.ar/ARTICULO ANUARIO.pdf

Barr, A. 1995. Management of Angular Limb Deformities in the Foal. Equine Veterinary Education. Vol 7 Num: 2: 75-78.

Baxter, G. \& Turner, S. 2004. Enfermedades de los huesos y las estructuras relacionadas. En: Adams. Claudicación en el caballo. Inter.-Médica, Buenos Aires, Argentina. p 407-423.

Carlson, C. L. Cullins, \& D. Meuten. 1995. Osteochondrosis of the Articular-Epiphyseal Cartilage Complex in Youngs Horses: Evidence for a Defect in Cartilage Canal Blood Supply. Vet. Pathol, 32:641- 647.

Clutterbuck A., P. Harris, D. Allaway, A. Mobasheri. 2010. Matrix metalloproteinases in inflammatory pathologies of the horse. Veterinary Journal 183 (1): 27-38.

Cruz, M. 2007. Fisiopatología de la osteocondritis disecantes en el caballo. www.produccion-animal.com.ar Foerner, J. 2003. Osteochondrosis in the Horse. Journal of Equine Veterinary Science, Vol 23, n. 4, 142 145.

Garner, L., J. Hiatt. 2002. Cartílago y hueso. Atlas de histología. Segunda edición. Mc Graw Hill. Interamericana. p.126-151.

Garzón Alvarado, D., M. Roa Garzón, A. Ramírez Martínez. 2008. Factores que influyen en el crecimiento endocondral: experimentos y modelos. Revista Cubana Ortopedia y Traumatología, Vol. 22.

Gandarillas M. (2008). Desórdenes ortopédicos en equinos: el factor nutricional. Agronomía y forestal № 35.Pp 30-34. http://www.uc.cl/agronomia/c extension/Revista/Ediciones/35/equino.pdf

Gaughan, E. 1988. Angular limb Deformities in Horses. The Compendium. Vol. 20(8) Page 944. Citado por Donald, E., Thrall, Manual de Diagnóstico Radiológico Veterinario. España. 4ta. Ed. Elsevier. p. 239.

Harris, P. 2005. Efecto de la nutrición y otros factores para asegurar un crecimiento óptimo en el potro. Waltham Centre for Pet Nutrition.

Matamala, G. 1999. Estudio de la casuística en equinos con afecciones del aparato locomotor, pacientes del Hospital Veterinario de la Universidad Austral de Chile durante los años 1976 a 1997. Tesis de grado no publicada, Universidad Austral de Chile. p.77

Mcllwraith, W. 2004. Developmental Orthopedic Disease: Problems of Limbs in Young Horses. Veterinary Review, 24(11):475-479.

Mericq, V. 2007. Factores reguladores de la osificación endocondral. Revista Médica Clínica Las Condes 18(4): 325-329.

Mitten L, \& A. Bertone. 1994. Angular limb deformities in foals. Department of Veterinary Clinical Sciences, Ohio State University. 204:717-20.

Novales, M. (2008). Sistema de control de la osteocondrosis en caballos de pura raza española. Revista electrónica de veterinaria Redvet. Vol. III, № 2, p.6.

O'Gradey, S. 2006. Flexural Deformities in Foals. Orthopaedic Research Center. Northern Virginia Equine. http://www.equineortho.colostate.edu/questions/flexural.htm.

Pagan, J. 2003. The Role of Nutrition in Developmental Orthopedic Disease: Nutritional Management. Diagnosis and Management of Lameness in the Horse. p 543-548.

Peralta, M. \& R. Batalla. 2009. Osteocondrosis en equinos.

http://www.engormix.com/MA-equinos/sanidad/articulos/osteocondrosis- equinost2734/165-p0.htm

Ralston, S. 2007. Evidence-Based Equine Nutrition. Veterinary Clinics of North America. Equine Practice 23 (2):365-384. 
Reca, M. 2005. Developmental orthopedic disease (DOD) and mare nutrition. The Horse.com Rooney, J. 1996. Locomotor System. In: Equine pathology. Ames, lowa. p 144-151 Sellnow, L. 2009. Alimentando al potro en crecimiento. The Horse.com. http://www.thehorse.com/articles/24569/thehorse-com-en-espantilde-ol-alimentando-al-potro-encrecimiento

Semevolos, S. \& A. Nixon. 2007. Osteochondrosis: Etiologic Facttors. Compendium: Equine Edition, article 2, $158-164$.

Silva Pérez, J. 2005. Prevalencia de las deformaciones angulares de los miembros en potrillos. Un estudio en cinco haras. Tesis de grado. Universidad Austral de Chile, Valdivia, Chile. p. 87.

Stephen, G., Jackson and J. D. Pagan. 2007. Developmental Orthopedic Disease: Multiple causes No sure cures. Journal of Equine Veterinary Science 22:37-45.

Thompson, K. 2007. Bones and joints. In: M.G. Maxie (Eds.). Jubb, Kennedy, and Palmer's Pathology of Domestic Animals: Volume 1. (5th Ed.) Edinburgh, UK: Elsevier Saunders. p. 1-184.

Trotter Gayle. W. 2006. Flexural Deformities. Orthopaedic ResearchCenter. Colorado State University. http://www.equineortho.colostate.edu/questions/flexural.htm

Van Weeren, R. 2006. Etiology, Diagnosis, and Treatment of OC. Clinical Techniques in Equine Practice. Vol. 5, Issue 4, 248-258.

Wagner P., G. Shires, B. Watrous, A. Kaneps, W. Schmotzer, \& T. Riebold. 1985. Management of acquired flexural deformities of the metacarpophalangeal joint. J Am Vet Med Assoc. 1; 187(9)915-8.

Wittwer, C. H. Hamann, E. Rosenberger, \& O. Distl. 2006. Prevalence of Osteochondrosis in the Limb Joints of South German Coldblood Horses. J. Vet. Med. A 53:531- 539.

Wyn-Jones, G. 1992. Enfermedades Ortopédicas de los Equinos. Cap. 7. Ed. Hemisferio Sur. p.160-174.

Ytrehus, B., C. Carlson, \& S. Ekman. 2007. Etiology and pathogenesis of osteochondrosis. Veterinary Pathology online 44:429-448. 